\title{
The Space Debris Environment: Future Evolution
}

\author{
Hugh G. Lewis, Graham G. Swinerd and Rebecca J. Newland
}

\author{
School of Engineering Sciences \\ University of Southampton \\ Southampton
}

SO17 1BJ, UK

\begin{abstract}
Space debris represents a significant risk to satellite operations, due to the possibility of damaging or catastrophic collisions. Consequently, many satellite operators screen the orbiting population for close approaches with their on-orbit assets and a public conjunction assessment service, Satellite Orbital Conjunction Reports Assessing Threatening Encounters in Space (SOCRATES), generates close approach predictions on a daily basis for all satellite payloads in the catalogue. These screening capabilities are used to inform operational decisions relating to risk mitigation but it is anticipated that the demands placed on these services will increase as debris becomes more prolific. This hypothesis is explored in a preliminary analysis of conjunction data for the years 2004 to 2009 and a new 'Business As Usual' study using the Debris Analysis and Monitoring Architecture for the Geosynchronous Environment (DAMAGE) model. The results suggest a 50\% increase in the number of close approaches reported by SOCRATES (or its equivalent) within the next ten years. By 2059, daily conjunction reports could contain over 50,000 close approaches below $5 \mathrm{~km}$, affecting the demands placed on tracking facilities and satellite resources.
\end{abstract}




\section{Introduction}

Space debris is now widely known to represent a significant risk to satellite operations, particularly in the low Earth orbit (LEO) region. Approximately 19,000 objects larger than 10 $\mathrm{cm}$ are known to exist, whilst the population of particles between 1 and $10 \mathrm{~cm}$ is estimated to be 500,000 and the number of smaller particles likely exceeds tens of millions (Orbital Debris Frequently Asked Questions available at http://orbitaldebris.jsc.nasa.gov/faqs.html). The sources of this debris population are space launches, on-orbit operations and fragmentations, with the latter accounting for over half of all the trackable objects in the current catalogue (Fig. 1).

\section{[ INSERT FIG.1 ABOUT HERE ]}

Debris mitigation measures identified by the Inter-Agency Space Debris Coordination Committee (IADC) and endorsed by the United Nations Committee on the Peaceful Uses of Outer Space (COPUOS) are already being implemented by space-faring nations. Key measures include de-orbiting spacecraft at the end of their mission, and removing stored energy by venting pressurised tanks and discharging batteries. Whilst these measures have succeeded in reducing the growth in the population of rocket bodies and mission-related debris since 2005 (gold and green curves in Fig. 2), recent fragmentation events, including the intentional destruction of the Fengyun-1C satellite in January 2007, have resulted in a significant increase in the number of objects larger than $10 \mathrm{~cm}$ (Fig. 2).

[ INSERT FIG. 2 ABOUT HERE ] 
As a result of historical space operations and fragmentations, and despite the adoption of several mitigation measures, it is likely that the population of orbiting objects has reached a critical point whereby growth will continue even in the absence of future space launches ${ }^{(1)}$. The basis for this growth comes from a widely-anticipated increase in the number of collisions involving large, intact objects. The remediation of the near-Earth environment, i.e. the removal of debris, is now accepted as the only solution to this problem ${ }^{(1)}$.

In order to better understand how recent trends in launch activity and fragmentations may affect the future evolution of the debris environment, the University of Southampton's Debris Analysis and Monitoring Architecture for the Geosynchronous Environment (DAMAGE) has been used in a new study of the 2009 to 2059 debris environment. The projection represents a revised 'Business As Usual' (BAU) scenario that assumes recently adopted mitigation measures will continue to be implemented and launch and explosion rates of the last decade will remain relevant for the next half-century. In addition, a study of close approach data from the NASA Goddard Space Flight Centre (GSFC), the European Space Operations Centre (ESOC), and the Centre for Space Standards and Innovation (CSSI) covering the period 2004 through 2009 has been undertaken. The purpose of the latter study is to provide some context to the future predictions made by the DAMAGE code. In particular, this paper focuses on the possible implications for close approach warnings, tracking requests and spacecraft collision mitigation manoeuvres.

\section{Study of Recent Collision and Close Approach Data}

\subsection{Overview}

A sign of the future collision activity was provided in February 2009, when the first collision between two intact spacecraft took place. The Iridium 33 - Cosmos 2251 event is only the 
most recent collision involving trackable orbiting objects. The European Space Agency’s Database and Information System Characterising Objects in Space (DISCOS) describes the occurrence of five historical 'natural' collisions since 1991, although Wright suggests that four additional collisions may have taken place (Fig. 3) ${ }^{(2)}$. Both 'histories' demonstrate a nonlinear (quadratic) increase in the number of collisions over time.

\section{[ INSERT FIG. 3 ABOUT HERE ]}

Whilst it is apparent that the time between collisions is of the order of years, orbiting objects experience many close approaches (with non-zero collision probability) over relatively short periods of time. For example, in 1996, the Computation of Miss Between Orbits (COMBO) program operated by the United States Space Control Center (SCC) revealed well over half a million close approaches under $100 \mathrm{~km}$ between catalogued objects per day ${ }^{1}$ (3). Approximately 1\% of these were below $10 \mathrm{~km}$ (Tab. 1). By 2005, the number of objects in the public catalogue had increased by 4\% from 9,556 in July 1996 to 9,941 in January 2005 (the month of the Thor Burner rocket body collision) and the number of close approaches below $100 \mathrm{~km}$ detected by COMBO had risen by 13\% to nearly three-quarters of a million per $\mathrm{day}^{2}$, with a corresponding increase in the number below $10 \mathrm{~km}^{(4)}$. Given the substantial growth in the number of catalogued objects since 2005, it is likely that the number of close approaches below $100 \mathrm{~km}$ involving catalogued objects now exceeds two million per day.

\section{[ INSERT TAB. 1 ABOUT HERE ]}

\footnotetext{
${ }^{1}$ On two randomly selected days in 1996 and the 24 hours before the CERISE/Ariane debris collision.

${ }^{2}$ On two randomly selected days in 2005 and the 24 hours before the Thor Burner rocket body collision.
} 


\section{[ INSERT FIG. 4 ABOUT HERE ]}

In addition to the analyses performed by the US SSC, the CSSI provide twice-daily reports on projected on-orbit conjunctions for the week ahead. These Satellite Orbital Conjunction Reports Assessing Threatening Encounters in Space (SOCRATES) ${ }^{(5)}$ list all close approaches below $5 \mathrm{~km}$ involving active and inactive satellite payloads using a maximum probability method $^{(6)}$. Reports from the last five years show a clear rise in the number of close approaches involving satellite payloads from approximately 7,000 in 2005 to nearly 14,000 today (Fig. 4). Whilst a significant number of these involve conjunctions with other spacecraft, the reports also demonstrate the impact that fragmentation events have had on the collision hazard faced by satellites operating in LEO: of the 14,828 close approaches predicted for the week 15 August - 22 August 2009, nearly half involved debris from recent major fragmentation events (Fig. 5).

\section{[ INSERT FIG. 5 ABOUT HERE ]}

When seen at a finer temporal resolution (Fig. 6), the number of weekly close approaches recorded in SOCRATES is strongly influenced by the number of debris added by fragmentation events. Here, data on all fragmentation events since November 2005 from ESA's DISCOS was used, together with catalogue data for recent, major fragmentation events reported by $\mathrm{NASA}^{(7)}$, and SOCRATES reports for the $15^{\text {th }}$ of each month from November 2005 to August 2009.

[ INSERT FIG. 6 ABOUT HERE ] 
Of considerable interest is the finding that the Iridium 33 - Cosmos 2251 collision and the Fengyun-1C event appear to be have had very similar effects on the number of close approaches experienced by satellites in the catalogue (an increase of approximately 3,000), even though the number of fragments generated by these events differed by $50 \%$. This may be due to the generation of two distinct debris clouds following the later collision, allowing fragments to interact with a wider range of orbits. Nearly $80 \%$ of all close approaches in recent SOCRATES reports involve debris. It is not surprising, therefore, that when the number of fragments generated by breakup events is used as an estimate of the number of close approaches (with the contribution of fragments generated by the Iridium 33 - Cosmos 2251 collision being doubled) the correlation is high $\left(R^{2}=0.88\right)$.

After accounting for the role played by fragmentation events in the close approaches reported from November 2005 to August 2009, a small increase due to the addition of new satellite payloads, rocket bodies and mission-related debris remained. This increase was found to be proportional to the number of intact objects on-orbit $\left(R^{2}=0.005\right.$ only) and equated to one additional close approach for every two intact objects added.

\subsection{Earth Science Satellite Data}

The computation of a collision probability requires knowledge of the uncertainty in the positions of the objects at the point of closest approach ${ }^{(8)}$. The magnitude of this uncertainty, arising from measurement errors and errors in the models used to propagate the orbits from the last measurement, determines the magnitude of the collision probability; a large positional uncertainty results in a small probability ${ }^{(6)}$. Thus, operational decisions, which could ultimately lead to a risk mitigation manoeuvre, often begin with requests for additional tracking data aimed at reducing the combined positional uncertainty. To understand the 
impact of close approach screening and the associated uncertainty on these operational decisions, a subset of the orbiting satellite population was studied in finer detail. The satellites chosen represent the foundation of recent Earth observation missions and several have been manoeuvred to avoid possible collisions.

The key driver for the future growth of the debris population has been identified as collision activity occurring in the $800-1000 \mathrm{~km}$ altitude regime ${ }^{(1)}$. Earth science satellites are typical residents of this region of LEO and regular close approach screening is performed for these spacecraft in order to minimise the risks posed by debris. The European Space Agency operates two environmental remote sensing spacecraft at $785 \mathrm{~km}$, Envisat and ERS-2, and NASA, together with other agencies, operates eleven spacecraft in the Morning and Afternoon Earth Science Constellations (ESCs) at altitudes between $600 \mathrm{~km}$ and $700 \mathrm{~km}$ (Tab. 2).

Close approaches involving the satellites in Tab. 2 were identified in SOCRATES reports for November 2005 through August 2009. Weekly averages for the NASA and ESA satellites were computed and were found to show a substantial rise over the study period (Fig. 7). Since 2006, satellites in the NASA ESCs have experienced an increase of $115 \%$ in the number of close approaches below $5 \mathrm{~km}$ in SOCRATES reports, and the ESA satellites have seen an increase of $170 \%$.

[ INSERT TAB. 2 ABOUT HERE ]

[ INSERT FIG. 7 ABOUT HERE ] 
At a finer temporal resolution, Fig. 8 and Fig. 9 show that links exist between the number of close approaches reported in SOCRATES involving the satellites in Tab. 2, the number of debris generated by recent fragmentation events (NASA: $R^{2}=0.65$, ESA: $R^{2}=0.22$ ), and the number of fragments from these events remaining on-orbit (NASA: $R^{2}=0.70$, ESA: $R^{2}=$ 0.28). Inspection of Fig. 8 and Fig. 9 reveals that, on average, every 1000 fragments (or every 500 fragments from the Iridium 33 - Cosmos 2251 collision) added to the orbiting population during the study period resulted in one extra close approach per satellite. Intact objects were often recorded in close approaches involving these Earth science satellites, at a ratio of approximately one close approach per satellite to every 4,000 intact objects in the catalogue, but the study was unable to determine this relationship reliably.

[ INSERT FIG. 8 ABOUT HERE ]

[ INSERT FIG. 9 ABOUT HERE ]

NASA Goddard Space Flight Center (GSFC) maintains its own close approach screening capability to protect the satellites in the $\operatorname{ESCs}^{(9)}$. Three safety volumes are used in the screening process and violations of these trigger different data product deliveries and actions. Tab. 3 lists the dimensions of these safety volumes and Fig. 10 shows the number of unique violations per year experienced by satellites in the constellations for the period 2005 through 2007.

[ INSERT TAB. 3 ABOUT HERE ] 
The number of ESC monitor volume violations rose from 5,133 in 2005 by $91 \%$ to 9,810 in 2007, and the number of tasking and watch violations show similar increases of approximately $100 \%$. The NASA monitor volume violation and SOCRATES data are reasonably correlated $\left(R^{2}=0.60\right)$, so it is likely that the factors influencing the number of close approaches below $5 \mathrm{~km}$ in SOCRATES are also important when considering the increase in safety volume violations. Figure 10 also shows the number of risk mitigation manoeuvres performed by the NASA ESC satellites in the years 2005 through 2007. Five manoeuvres were made during this period, with most taking place in 2007.

\section{[ INSERT FIG. 10 ABOUT HERE ]}

Close approach screening for ESA's Envisat and ERS-2 satellites is performed at the European Space Operations Centre. Logs of all conjunction events are maintained and the data for the period 2004 to 2009 are shown in Fig. 11. Warnings are triggered if a 1:10,000 collision probability threshold is exceeded. The mechanism to use tracking data was implemented in 2005 and became operational in 2006, significantly reducing the number of risk mitigation manoeuvres (Krag, personal communication). A total of five collision avoidance manoeuvres performed by Envisat and ERS-2 are shown in Fig. 11. However, tracking data has not always been available when desired due to non-availability of the radar facility or a short lead-time before the conjunction.

\section{[ INSERT FIG. 11 ABOUT HERE ]}

Figure 11 provides further evidence of the increasing threat to Earth science spacecraft. In particular, analysts at ESA ESOC noted that the number of high-risk conjunction events 
doubled on average following the Iridium 33 - Cosmos 2251 collision on 10 February 2009 (Krag, personal communication).

\subsection{Summary}

Based on the study of SOCRATES, NASA and ESA close approach data for the recent past, it is likely that the number of intact objects on-orbit and the number of large fragmentation events will significantly affect the number of close approaches experienced by satellite payloads in the future. Periods of steady growth in the population of orbiting objects, due to continuing launch activities and space operations, will be reflected in a small, but steady rise in the number of close approaches experienced by spacecraft. Analysis of SOCRATES data suggests that for every two intact objects added to the catalogue between November 2005 and August 2009, one additional close approach below $5 \mathrm{~km}$ was recorded. On average this equates to an increase of 0.06 close approaches for each of the approximately 3,000 active or inactive satellite payloads for every intact object added to the catalogue. However, the risk to satellites is not uniformly distributed; for the 13 Earth science satellites studied here, operating at altitudes between 600 and $700 \mathrm{~km}$, there is approximately one additional close approach per satellite for every 1000 intact objects added to the catalogue (i.e. six times higher than the average).

This steady growth in conjunctions will be punctuated by sudden, large increases following major fragmentation events (explosions or collisions), with the actual impact being affected by the type of event and location. Assuming the trends of the recent past continue into the future, the number of additional close approaches in the SOCRATES reports introduced by a breakup will be approximately equal to the number of fragments generated. For catastrophic collisions involving two intact objects, the number of additional close approaches may be 
twice the number of fragments generated. For example, fragments from the Iridium $33-$ Cosmos 2251 collision, for example, have been responsible for approximately 3,000 additional close approaches per week, since February 2009. The increase was similar following the intentional breakup of Fengyun-1C.

The additional close approaches equate to a real increase in risk to these satellites, as the collision probability shows an equivalent increase over the study period (Fig. 12).

\section{[ INSERT FIG. 12 ABOUT HERE ]}

The increase in close approaches, whether due to continuing space activities or fragmentation events, will clearly influence the number of tracking requests and, ultimately, the number of risk mitigation manoeuvres performed. This will have significant implications for the Space Situational Awareness (SSA) capabilities and services planned by ESA. By combining the ESA close approach and SOCRATES data for the years 2006 to 2008, we see about one ESA tracking campaign per satellite for every 3,700 close approaches under $5 \mathrm{~km}$ identified in SOCRATES. Analysing the data from NASA and ESA for the years 2006 and 2007 reveals one manoeuvre for every 1,320 close approaches involving the 13 Earth science satellites identified in SOCRATES (i.e. approximately one manoeuvre per satellite for every 17,200 close approaches).

\section{DAMAGE Business As Usual Study}

\subsection{The DAMAGE model}

The University of Southampton's debris model, DAMAGE, is a three-dimensional model of the full low Earth orbit to geosynchronous orbit debris environment. As with other 
evolutionary models, DAMAGE is able to simulate the historical and future debris populations $\geq 10 \mathrm{~cm}$ using a Monte Carlo (MC) approach, whereby multiple projection runs are performed to establish reliable statistics. Projections covering the historical period from 1957 to 2009 employ launch and fragmentation information from ESA's DISCOS and historical monthly averaged solar flux F10.7 values combined with the CIRA-72 atmospheric model for atmospheric drag calculation (Fig. 13).

\section{[ INSERT FIG. 13 ABOUT HERE ]}

Future projections from August 2009 repeat launch and explosion events from the period August 1999 - August 2009 (excluding all collision events, and the breakups of Fengyun-1C and USA-193, but including one major explosion event per decade) to simulate future launch and explosion activity, with a long-term F10.7 projection. All fragmentation events are simulated using the NASA Standard Breakup Model ${ }^{(10)}$ with the parameters of historical fragmentation events selected to produce a good agreement with catalogue $\operatorname{data}^{3}$ (Fig. 13). Objects are propagated forwards using a semi-analytical orbital propagator that includes Earth's $J_{2}, J_{3}, J_{2,2}$, luni-solar gravitational perturbations, solar radiation pressure (with cylindrical Earth shadow) and atmospheric drag.

Collision probabilities are estimated using a fast, pair-wise algorithm based on the 'Cube' approach adopted in NASA's LEO-to-GEO Environment Debris model (LEGEND) ${ }^{(11)}$. Comparison with historical collision activity (Fig. 14) allows future predictions to be interpreted in the context of historical rates.

\footnotetext{
${ }^{3}$ Fewer than 100 fragments from the February 2007 breakup of a Briz-M second stage have been added to the catalogue, although it was suspected to have generated nearly 1000 fragments.
} 
[ INSERT FIG. 14 ABOUT HERE ]

Typically, 'Business As Usual' studies assume a 'worst case' scenario, whereby debris mitigation measures, including de-orbiting spacecraft at the end of their mission and passivation, are not implemented in the future projection. However, it is apparent from the historical evolution of the debris environment (Fig. 2) that mitigation measures have been having a positive impact on the number of orbiting rocket bodies and mission-related debris. Whilst unintentional fragmentation events continue to occur, it now appropriate to consider a more optimistic future for the BAU scenario. Consequently, we have adopted a BAU study that assumes a 50\% success rate for de-orbiting spacecraft and rocket bodies launched after 1 January 2007 (following the 25-year rule). Twenty Monte-Carlo runs of this new BAU scenario were used to estimate the number of objects and the number of collisions (using cubes of $20 \mathrm{~km}$ ) in the projection period 1 August 2009 to 1 August 2059.

\subsection{Results}

Fig. 15 shows the number of objects on-orbit, by type, for the period 2009 through 2059. The total number of objects increases from approximately 15,000 in 2009 to 23,500 in 2059, with intacts (spacecraft, rocket bodies and mission-related debris) accounting for approximately half, and collision fragments accounting for nearly one-third, of this increase.

\section{[ INSERT FIG. 15 ABOUT HERE ]}

The corresponding estimate of the number of collisions occurring in the projection period is shown in Fig. 16. Approximately seven catastrophic collisions (in which the ratio of impactor 
kinetic energy to target mass is greater than $40 \mathrm{~J} / \mathrm{g}$ ) occur in this time. It is important to note that whilst the number of collisions is fundamental to this study, the shapes of the curves in Fig. 16 are of great concern. These curves demonstrate a non-linear (quadratic) growth in the number of collisions, as seen in the historical data, even though the projected collision rate appears lower than the historical rate (Fig. 14). This indicates instability, even in the presence of debris mitigation, which will need to be addressed through remediation.

[ INSERT FIG. 16 ABOUT HERE ]

[ INSERT FIG. 17 ABOUT HERE ]

The DAMAGE projection suggests that within ten years the number of intact objects on-orbit will have risen by approximately 1000 (Fig. 15) in the BAU scenario. If the relationship between the number of intact objects on-orbit and the number of close approaches is maintained over this period, then this increase equates to 500 additional close approaches for satellite payloads per week. However, the projected fragmentation events will contribute approximately 6,000 new close approaches in the same time. Consequently, the total number of close approaches reported each day (for the week ahead) could be more than 21,000 in 2019, or 50\% more than it is today (Fig. 17). Clearly, A higher collision rate than the one predicted by DAMAGE will mean a substantially higher number of close approaches.

Satellites operating in the orbital regime used by the 13 spacecraft in Tab. 2 could each appear in the daily conjunction reports an additional seven times (i.e. 20 close approaches per satellite in total) by 2019. From the analysis of historical ESA and NASA risk mitigation 
data, results from the BAU projection suggest that the interval between tracking campaigns for each satellite will be 3.6 years and one collision avoidance manoeuvre will be required every 16.6 years by 2019 (Fig. 18).

The operational demands will increase in a non-linear manner, due to the increasing frequency of catastrophic collisions, such that within 50 years each daily conjunction report may contain over 50,000 close approaches under $5 \mathrm{~km}$ for satellite payloads (Fig. 17). By this time, satellites in high-risk orbital regimes may appear 50 times in each conjunction report, and the intervals between tracking campaigns and collision avoidance manoeuvres for each satellite will be nearly one-quarter of present day values (1.4 years and 6.4 years, respectively; Fig. 18).

[ INSERT FIG. 18 ABOUT HERE ]

\section{Conclusions}

Recent fragmentation events, including the intentional destruction of the Fengyun-1C satellite and the accidental collision of Iridium 33 and Cosmos 2251 have resulted in a $40 \%$ rise in the number of catalogued objects since 2005, with some orbital altitudes bands seeing an increase of $200 \%$ in spatial density. Fragments from these two events are currently responsible for nearly half of all close approaches involving satellite payloads recorded in SOCRATES. Space agencies operating Earth science spacecraft between 600 and $700 \mathrm{~km}$ altitude, and performing regular close approach screening, have seen the number of close approaches detected increase over the last fours years. For Envisat and ERS-2, ESA have observed as many close approaches in the first half of 2009 as were recorded in all of 2008, for example. 
Continued close approaches involving valuable space assets have implications for operational decision-making, which affect tracking campaigns and risk mitigation manoeuvres.

Previous studies of the future debris environment have often focused on the evolution of the gross characteristics, such as the number of objects or the number of collisions, without necessarily considering the effect the changing environment has on satellite operations. In this work, results from a DAMAGE BAU scenario have been used to estimate the impact that an increasing debris population might have on close approach screening results, and decisions relating to tracking campaigns and collision avoidance manoeuvres. The results suggest a $50 \%$ increase in the number of close approaches in the next ten years and a $250 \%$ increase in the next 50 years. Satellites and constellations of satellites, especially those in high-risk altitude bands, will require significant operational support as a consequence of these new close approaches. In spite of tracking campaigns to reduce uncertainty, each satellite is likely to require at least one collision avoidance manoeuvre in its operational lifetime by 2059 . Beyond 2059, the frequency of tracking campaigns and manoeuvres will increase, due to the non-linear growth of the number of catastrophic collisions.

\section{Acknowledgments}

We would like to extend our thanks to Lauri Newman (NASA GSFC), Holger Krag (ESA ESOC), Tim Payne (USAF), and T.S. Kelso (CSSI) for providing historical conjunction event and risk mitigation data, and J.-C. Liou (NASA JSC) for providing the historical catalogue data. 


\section{References}

1. Liou, J.-C., and Johnson, N.L. Risks in space from orbiting debris. Science, 311, 2006, pp. 340-341.

2. Wright, D. Colliding satellites: consequences and implications. Union of Concerned Scientists, 2009, http://www.ucsusa.org/nuclear_weapons_and_global_security/space_weapons/technic al_issues/colliding-satellites.html, accessed 28 September 2009.

3. Payne, T.P. First “confirmed” natural collision between two catalogued satellites. Proceedings of the Second European Conference on Space Debris, Darmstadt, Germany, March 17-19 1997.

4. Payne, T.P., Morris, R.F., Taft DeVere, G., Thurston, R.G., Ward, D.A., and Teets, R.B. The second confirmed unintentional on-orbit collision. Proceedings of the AMOS Technical Conference, Maui, Hawaii, September 2005.

5. Kelso, T.S., and Alfano, S. Satellite Orbital Conjunction Reports Assessing Threatening Encounters in Space (SOCRATES). Proceedings of the Fourth European Conference on Space Debris, Darmstadt, Germany, April 18-20 2005.

6. Alfano, S. Relating position uncertainty to maximum conjunction probability. The Journal of the Astronautical Sciences, 53, (2), 2005, pp. 193-205. 
7. Liou, J.-C. Update on recent major breakup fragments, Orbital Debris Quarterly News, 13, (3), 2009, pp. 5-6.

8. Ailor, W.H., and Peterson, G.E. Collision avoidance as a debris mitigation measure. 55th International Astronautical Congress, Vancouver, Canada, October 2004, IAC04-IAA.5.12.3.01.

9. Newman, L.K. The NASA robotic conjunction assessment process: overview and operational experiences. $59^{\text {th }}$ International Astronautical Congress, Glasgow, Scotland, October 2008, IAC-08-A.6.2.6.

10. Johnson, N.L., Krisko, P.H., Liou, J.-C., and Anz-Meador, P.D. NASA’s new breakup model of EVOLVE 4.0. Advances in Space Research, 28, (9), 2001, pp. 1377-1384.

11. Liou, J.-C., Hall, D.T., Krisko, P.H., and Opiela, J.N. LEGEND - a three-dimensional LEO-to-GEO debris evolutionary model, Advances in Space Research, 34, 2004, pp. 981-986. 


\section{Tables}

Table 1. Average number of close approaches per day identified by the COMBO program for the years 1996 and 2005 (data: Payne ${ }^{(3)}$ and Payne et $\mathrm{al}^{(4)}$ ).

\begin{tabular}{ccc}
\hline & $\mathbf{1 9 9 6}$ & $\mathbf{2 0 0 5}$ \\
\hline$<100 \mathrm{~km}$ & 631,766 & 713,739 \\
$<25 \mathrm{~km}$ & 42,281 & 40,292 \\
$<10 \mathrm{~km}$ & 6,864 & 7,814 \\
\hline
\end{tabular}

Table 2. NASA and ESA* operated Earth science satellites used in this study.

\begin{tabular}{lcc}
\hline \multicolumn{1}{c}{ Satellite } & Perigee & Apogee \\
& Altitude $\mathbf{( k m )}$ & Altitude $\mathbf{( k m )}$ \\
\hline AQUA & 702 & 703 \\
AURA & 702 & 703 \\
CALIPSO & 702 & 704 \\
CLOUDSAT & 702 & 704 \\
EO-1 & 680 & 694 \\
ICESAT & 595 & 596 \\
LANDSAT 5 & 701 & 705 \\
LANDSAT 7 & 702 & 703 \\
PARASOL & 702 & 703 \\
SAC C & 703 & 705
\end{tabular}


TERRA

ENVISAT $^{*}$

ERS 2*
702

784

784
704

785

785

Table 3. Safety volume definitions for NASA's ESC close approach screening ${ }^{(9)}$.

\begin{tabular}{lcccc}
\hline Safety & & Radial & In- & Cross- \\
volume & Shape & track & track \\
& & $(\mathbf{k m})$ & $\mathbf{( k m )}$ & $\mathbf{( k m )}$ \\
\hline Monitor & Ellipsoid & \pm 2 & \pm 25 & \pm 25 \\
Tasking & Box & \pm 0.5 & \pm 5 & \pm 5 \\
Watch & Sphere & & 1 & \\
\hline
\end{tabular}




\section{Figure Captions}

Figure 1. Objects in Earth orbit on 1 April 2009 by type (data: NASA).

Figure 2. Monthly number of objects in Earth orbit (data: NASA).

Figure 3. Historical collision activity (data: ESA DISCOS).

Figure 4. Average ${ }^{4}$ number of close approaches under $5 \mathrm{~km}$ per week involving satellite payloads for November 2005 through August 2009 (data: CSSI SOCRATES).

Figure 5. Contribution of recent breakup fragments to SOCRATES close approaches (data: CSSI SOCRATES, generated at 13:33 on 15 August 2009).

Figure 6. The influence of fragmentation debris on the number of weekly close approaches recorded in SOCRATES (for clarity, curves show the change since November 2005. Data: CSSI SOCRATES, ESA and NASA).

Figure 7. Average ${ }^{5}$ number of close approaches per week for November 2005 through August 2009 (data: CSSI SOCRATES).

\footnotetext{
${ }^{4}$ The SOCRATES data were sampled at monthly intervals and yearly averages were computed from these sampled values.

${ }^{5}$ The SOCRATES data were sampled at monthly intervals and yearly averages were computed from sampled values.
} 
Figure 8. The influence of fragmentation debris on the number of weekly close approaches recorded by SOCRATES for NASA ESC satellites (for clarity, curves show the change since November 2005. Data: CSSI SOCRATES, ESA and NASA).

Figure 9. The influence of fragmentation debris on the number of weekly close approaches recorded by SOCRATES for ESA's Envisat and ERS-2 satellites (for clarity, curves show the change since November 2005. Data: CSSI SOCRATES, ESA and NASA).

Figure 10. Number of unique NASA ESC safety volume violations and risk mitigation manoeuvres for 2005 through 2007 (data: NASA).

Figure 11. Total number of unique Envisat and ERS-2 volume violations and risk mitigation manoeuvres for 2004 through June 2009 (data: ESA).

Figure 12. Increase in collision probability (sum computed over seven days) and best-fit curve (power law) for NASA ESC satellites and ESA's Envisat and ERS-2 over the period November 2005 to August 2009 (data: CSSI SOCRATES).

Figure 13. Comparison of DAMAGE historical evolution with historical catalogue data (data: NASA).

Figure 14. Comparison of DAMAGE historical evolution with historical collision data (data: DISCOS and Wright ${ }^{(2)}$ ). 
Figure 15. Average number of objects on-orbit through the BAU projection period computed from 20 DAMAGE MC runs.

Figure 16. Average number of collisions through the BAU projection period computed from 20 DAMAGE MC runs.

Figure 17. Average number of close approaches per week for the BAU projection computed from 20 DAMAGE MC runs.

Figure 18. Average number of close approaches per Earth science satellite per week for the BAU projection computed from 20 DAMAGE MC runs. The intervals between collision avoidance manoeuvres and tracking campaigns are also shown. 
Fig. 1

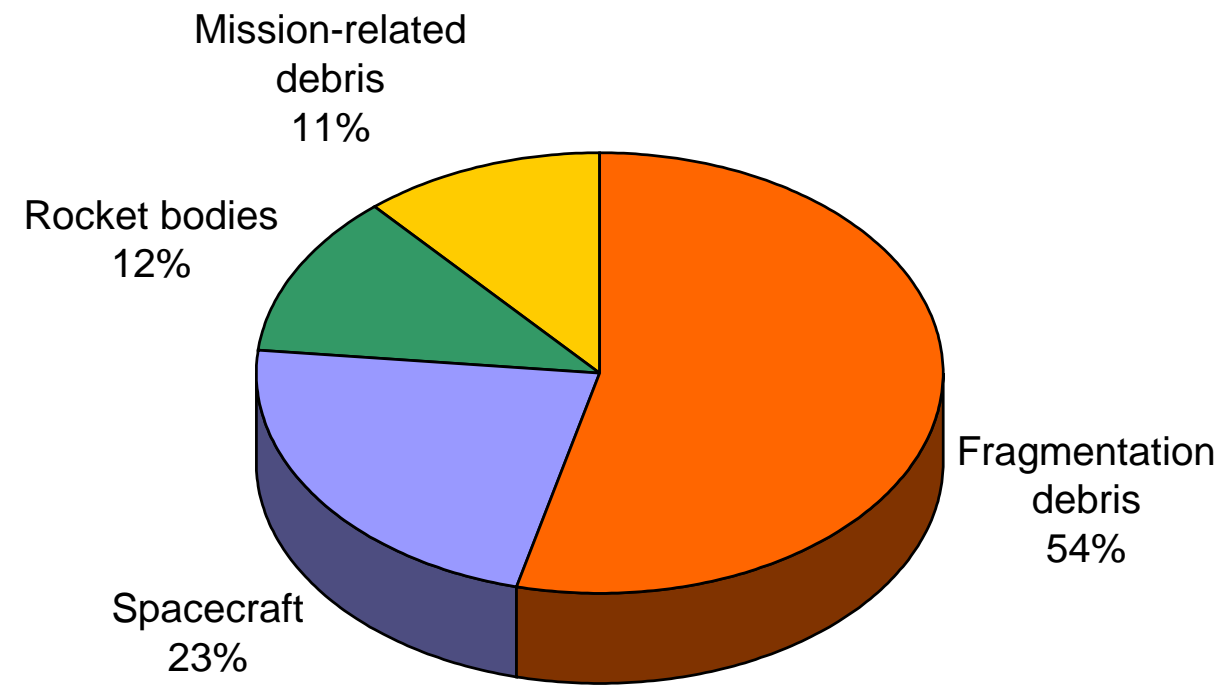


Fig. 2

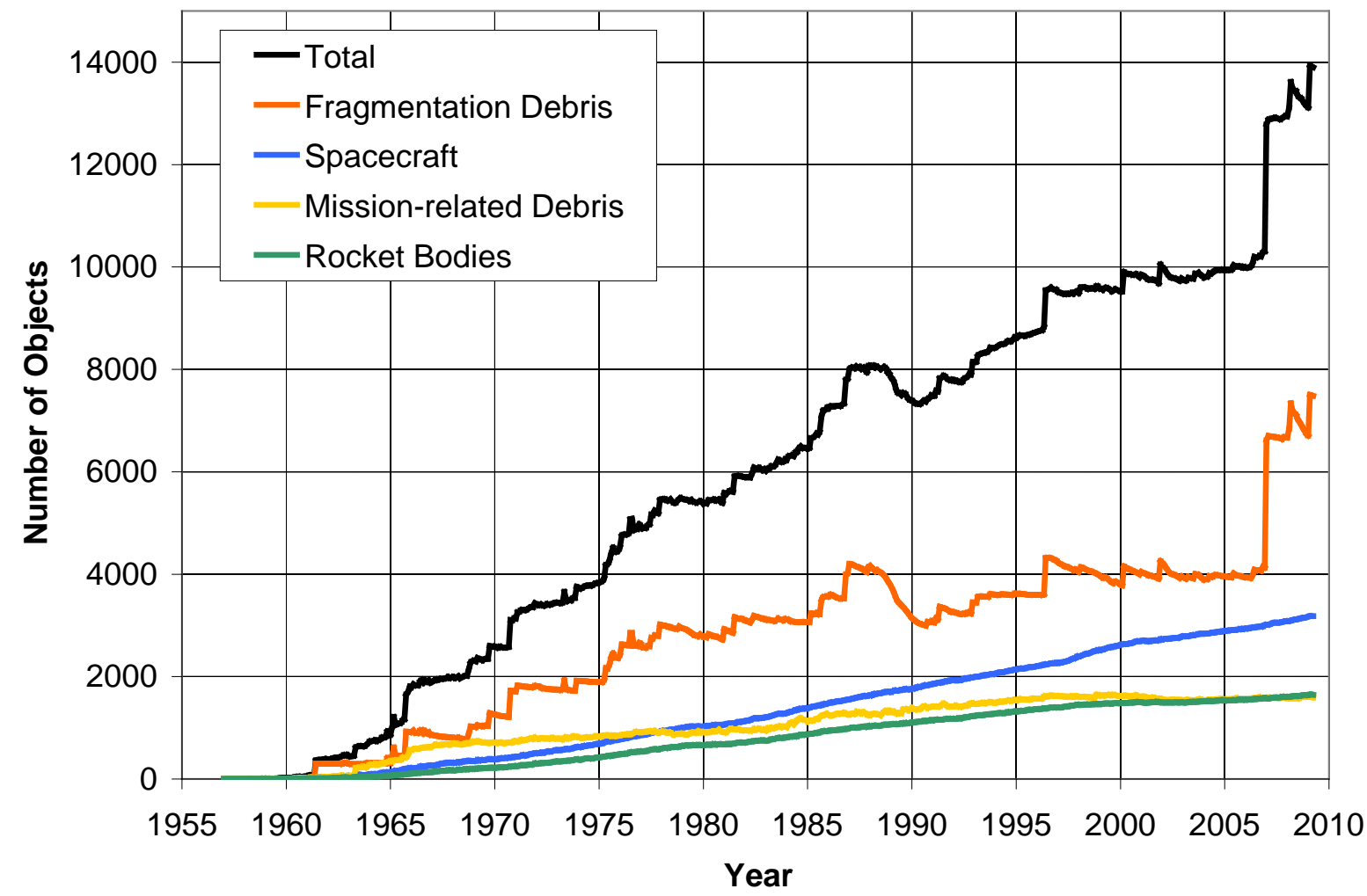


Fig. 3

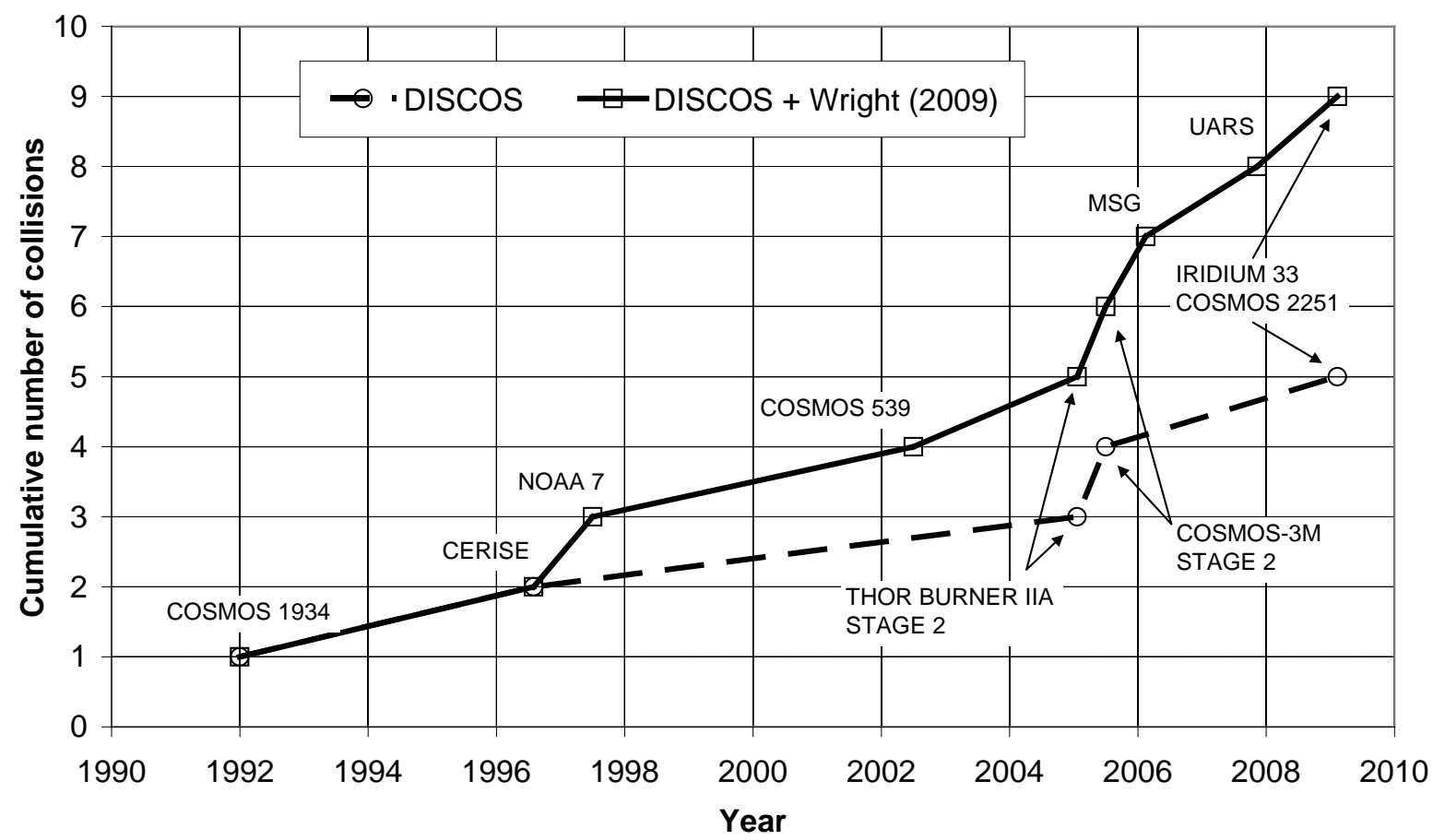


Fig. 4

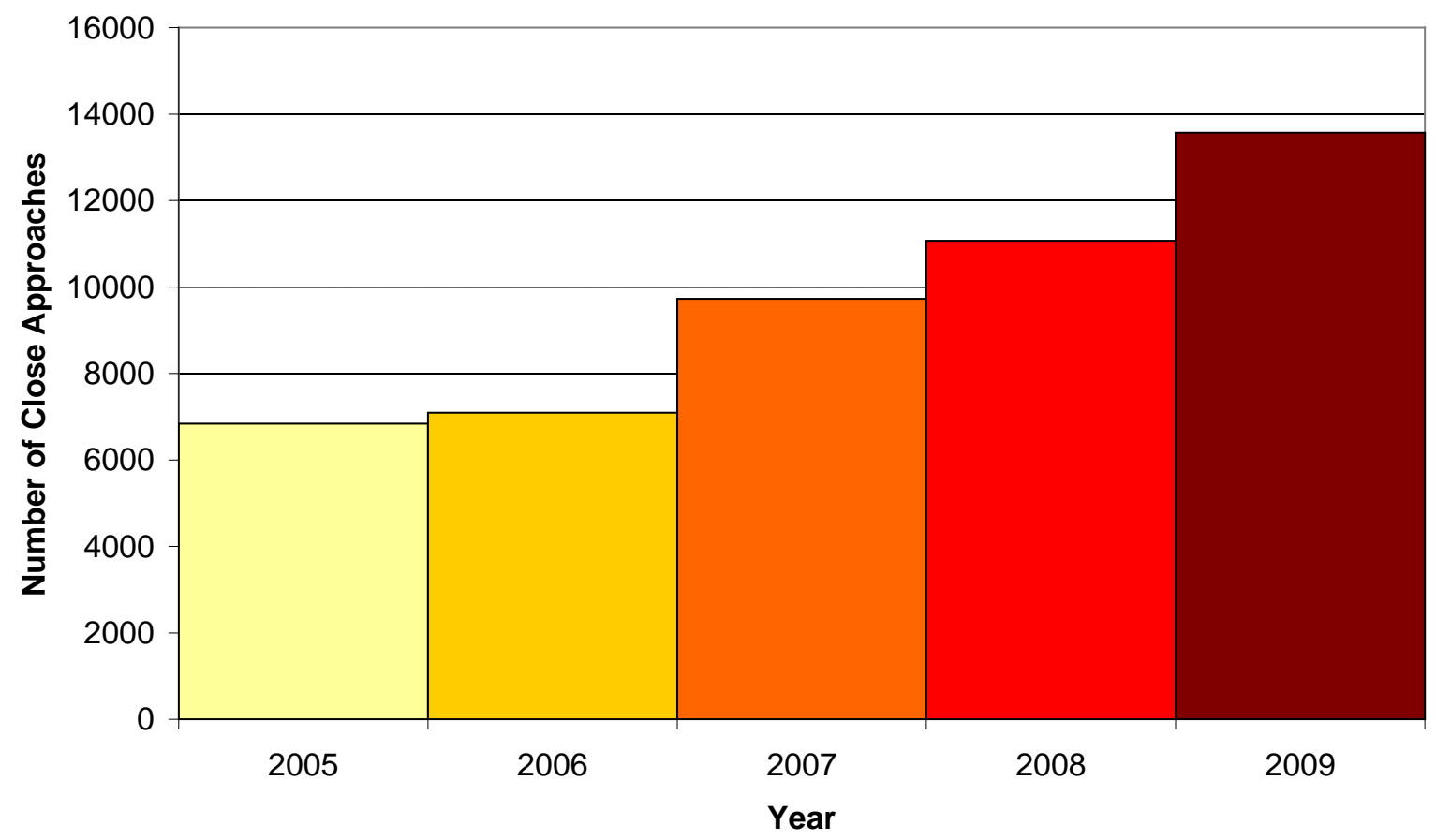


Fig. 5

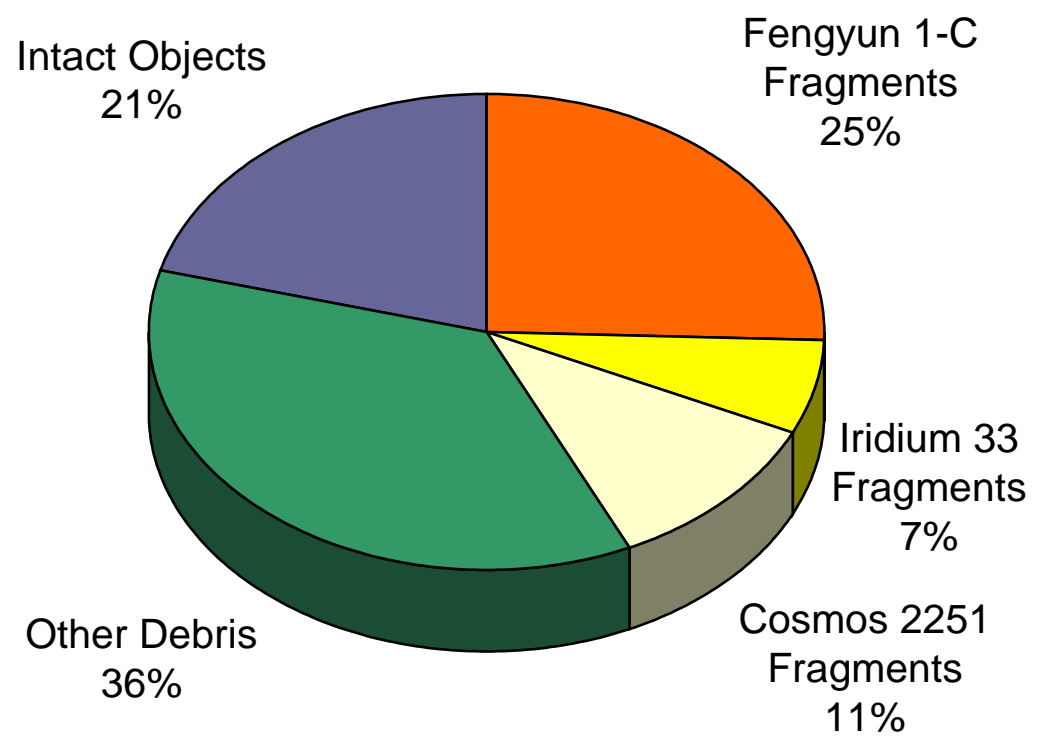


Fig. 6

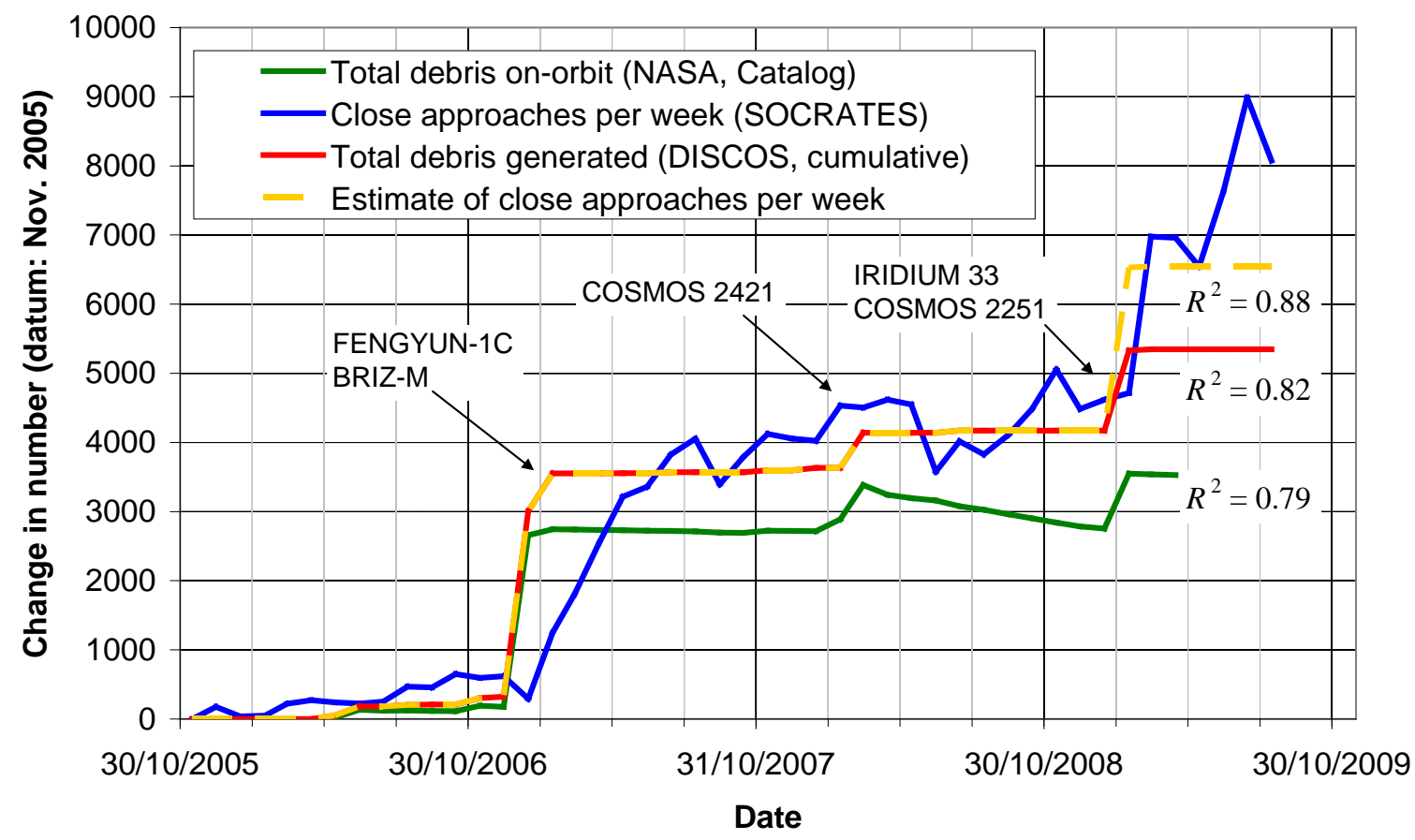


Fig. 7

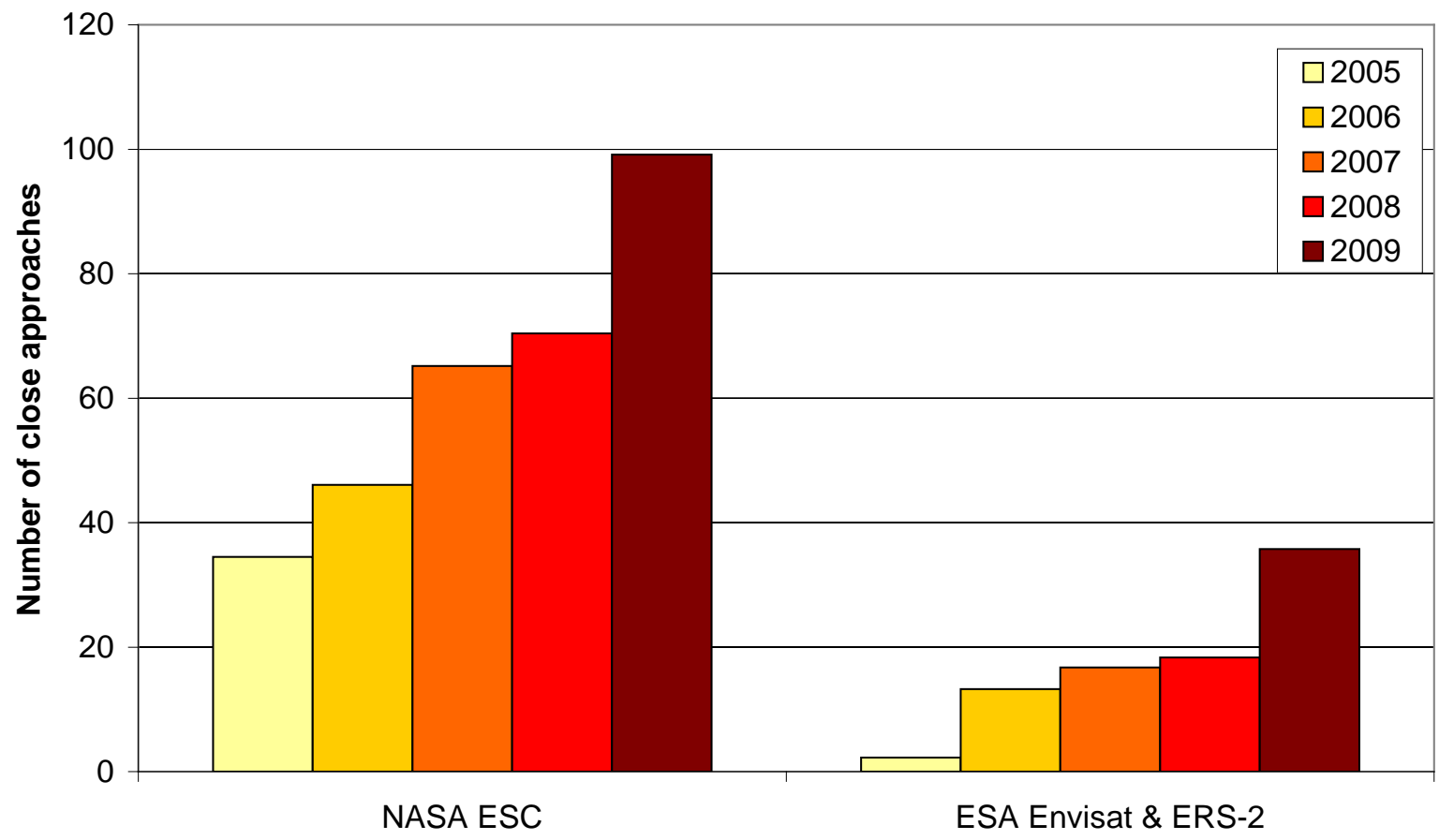


Fig. 8.

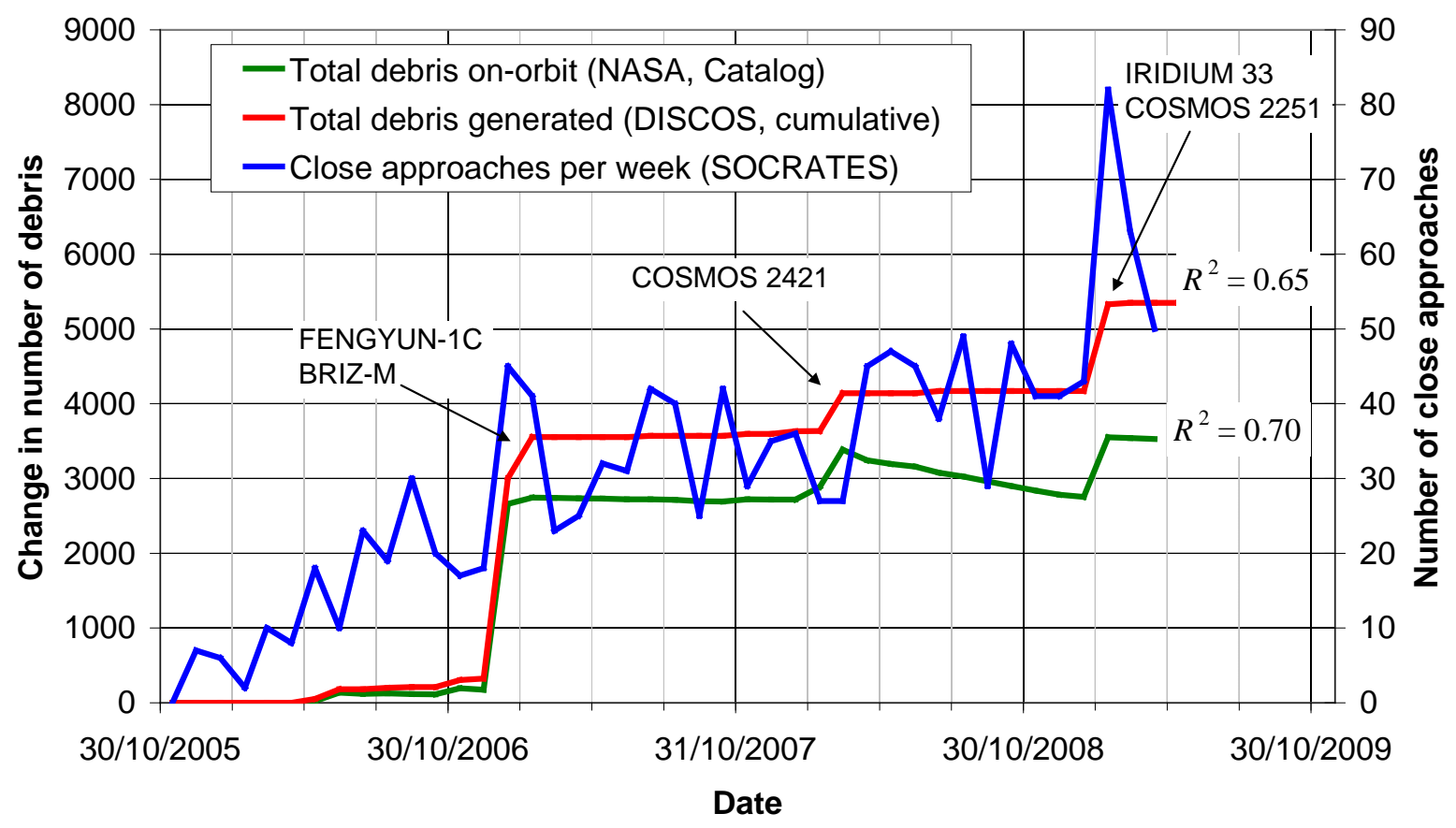


Fig. 9

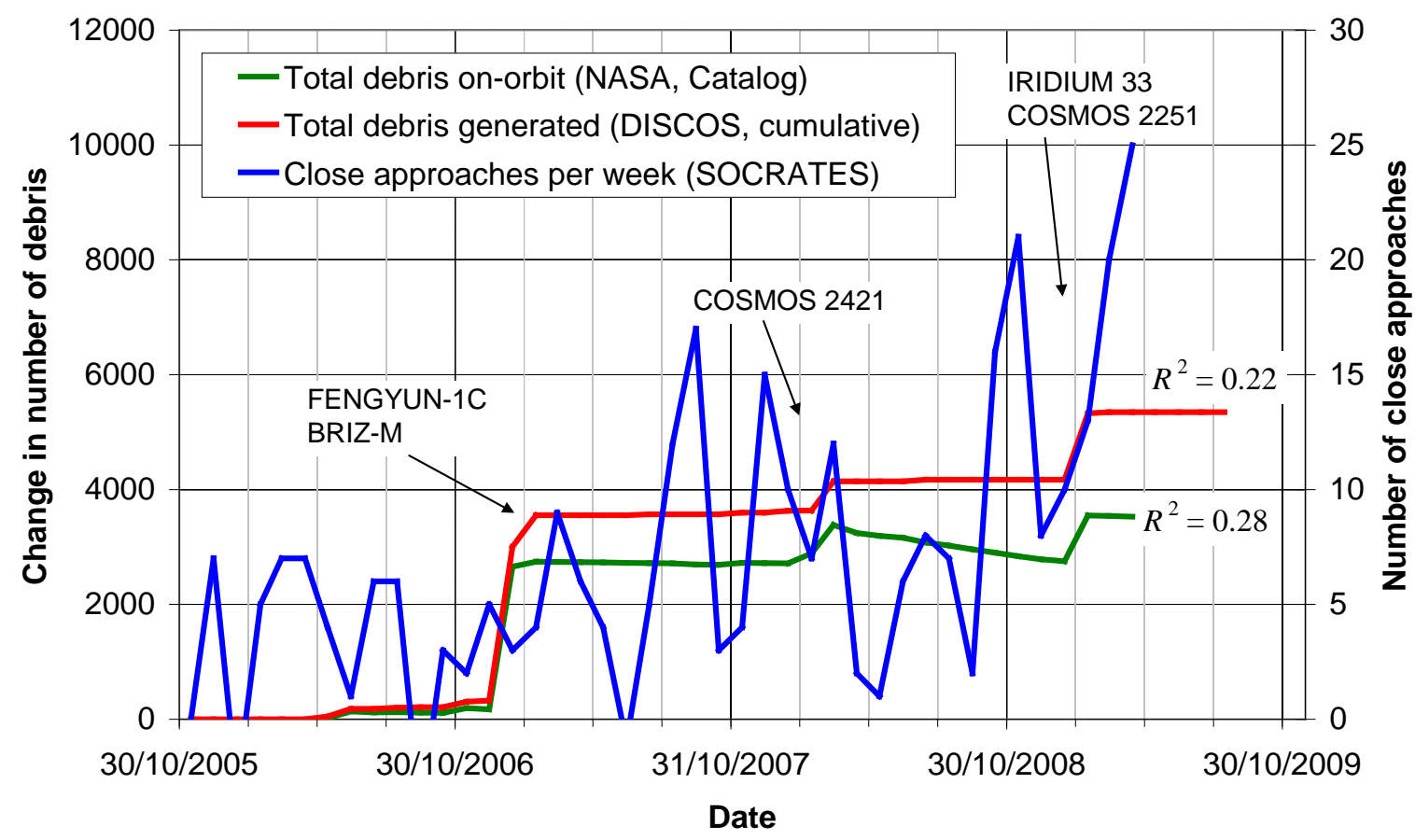


Fig. 10

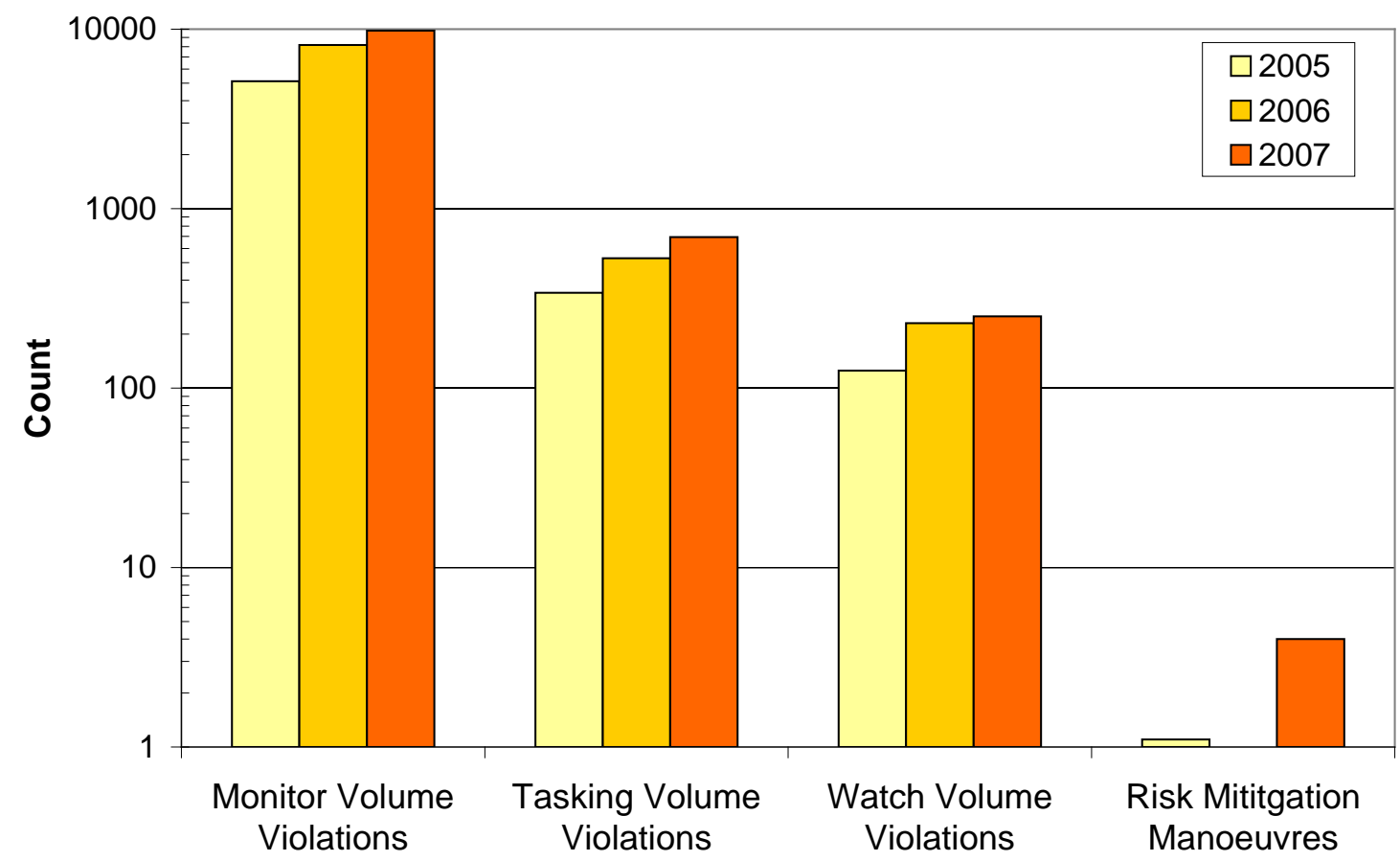


Fig. 11

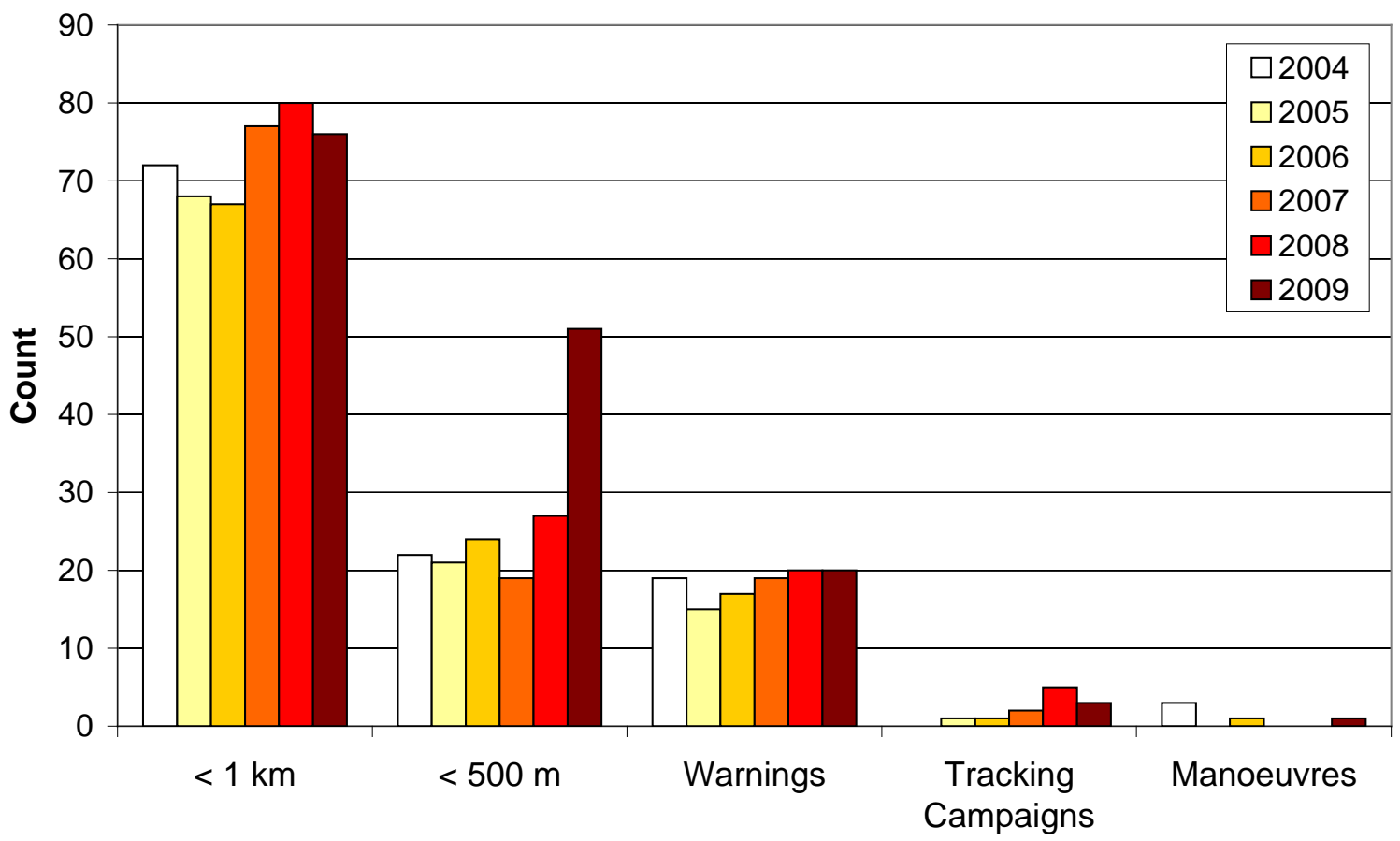


Fig. 12

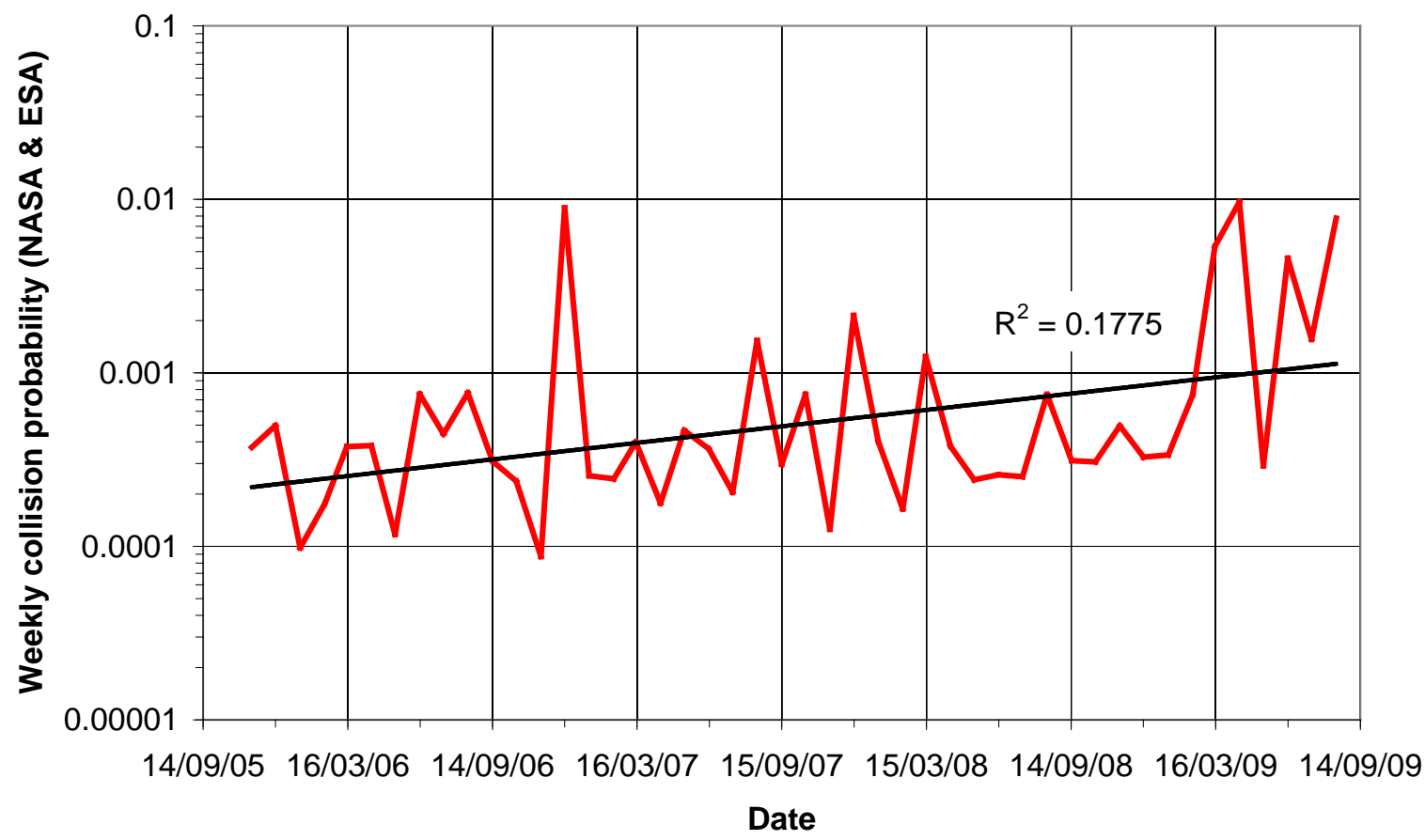


Fig. 13

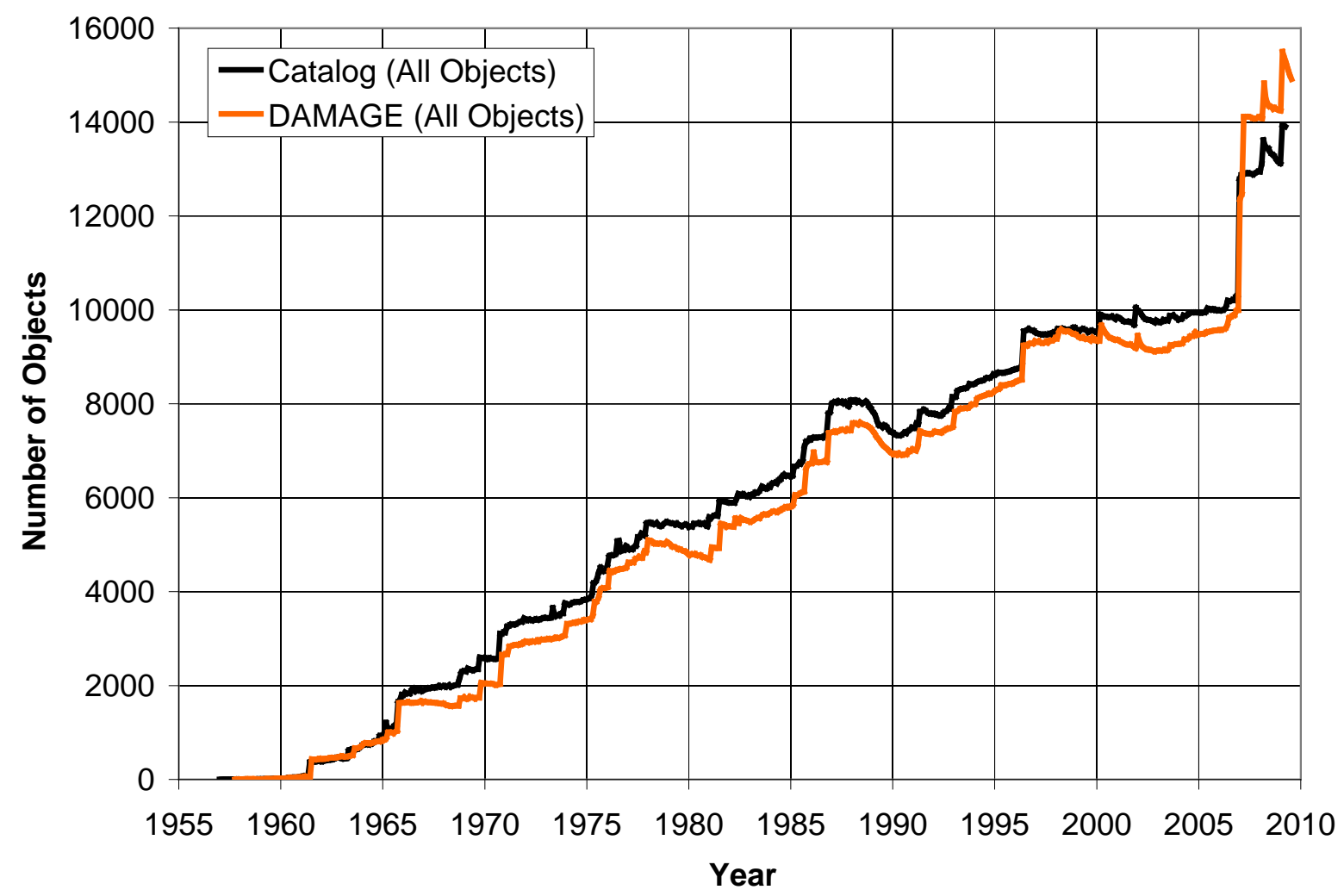


Fig. 14

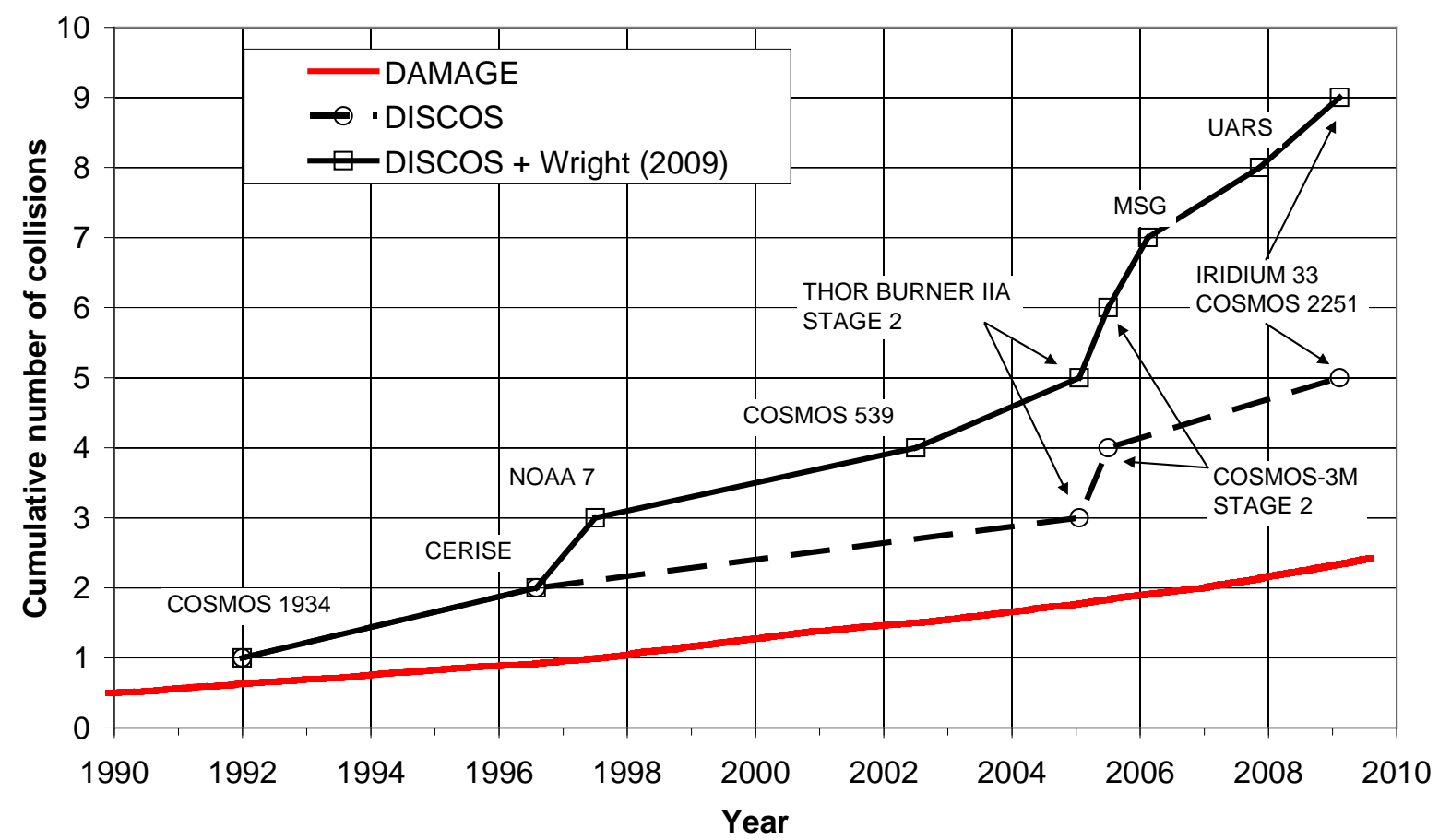


Fig. 15

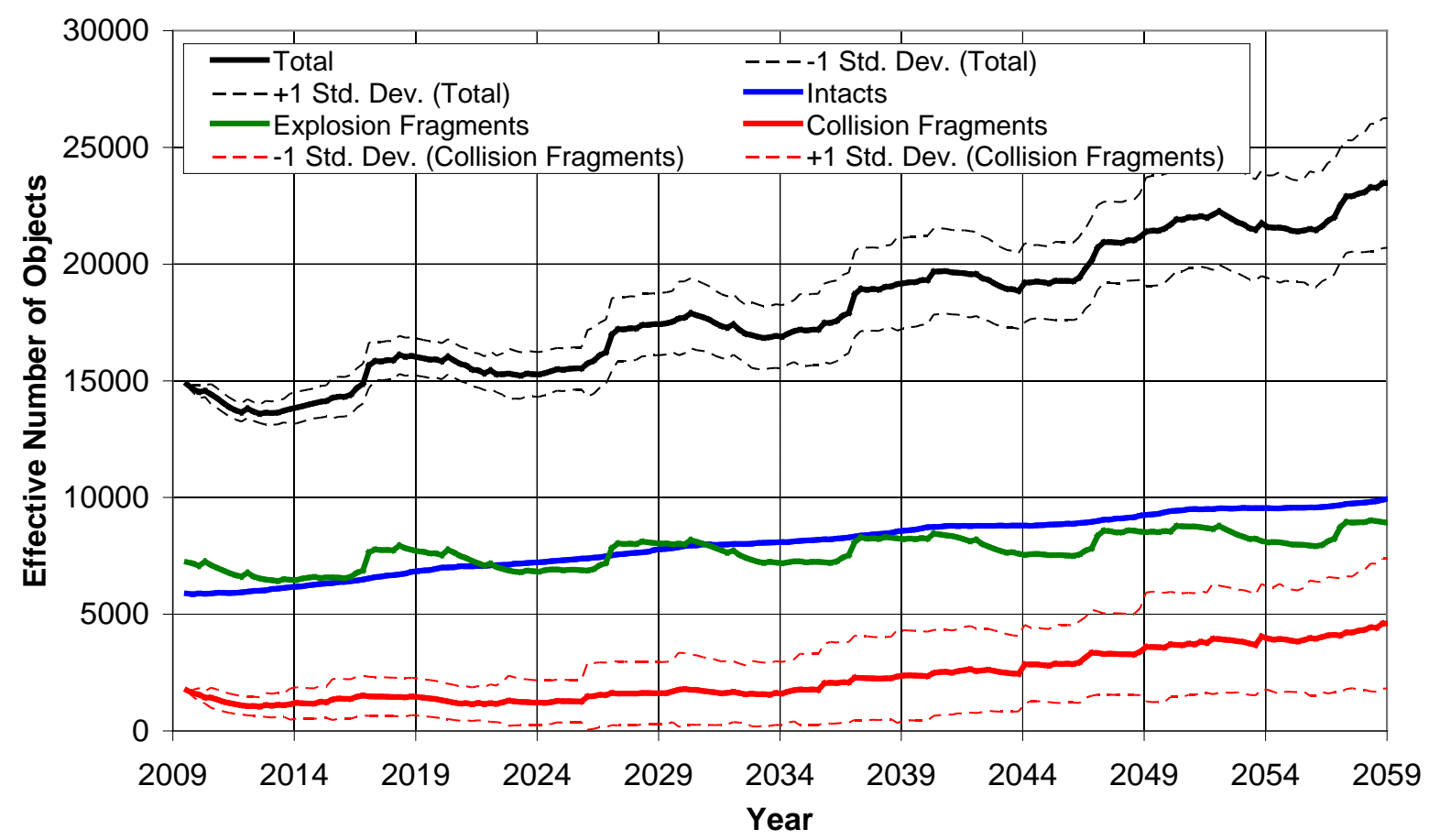


Fig. 16

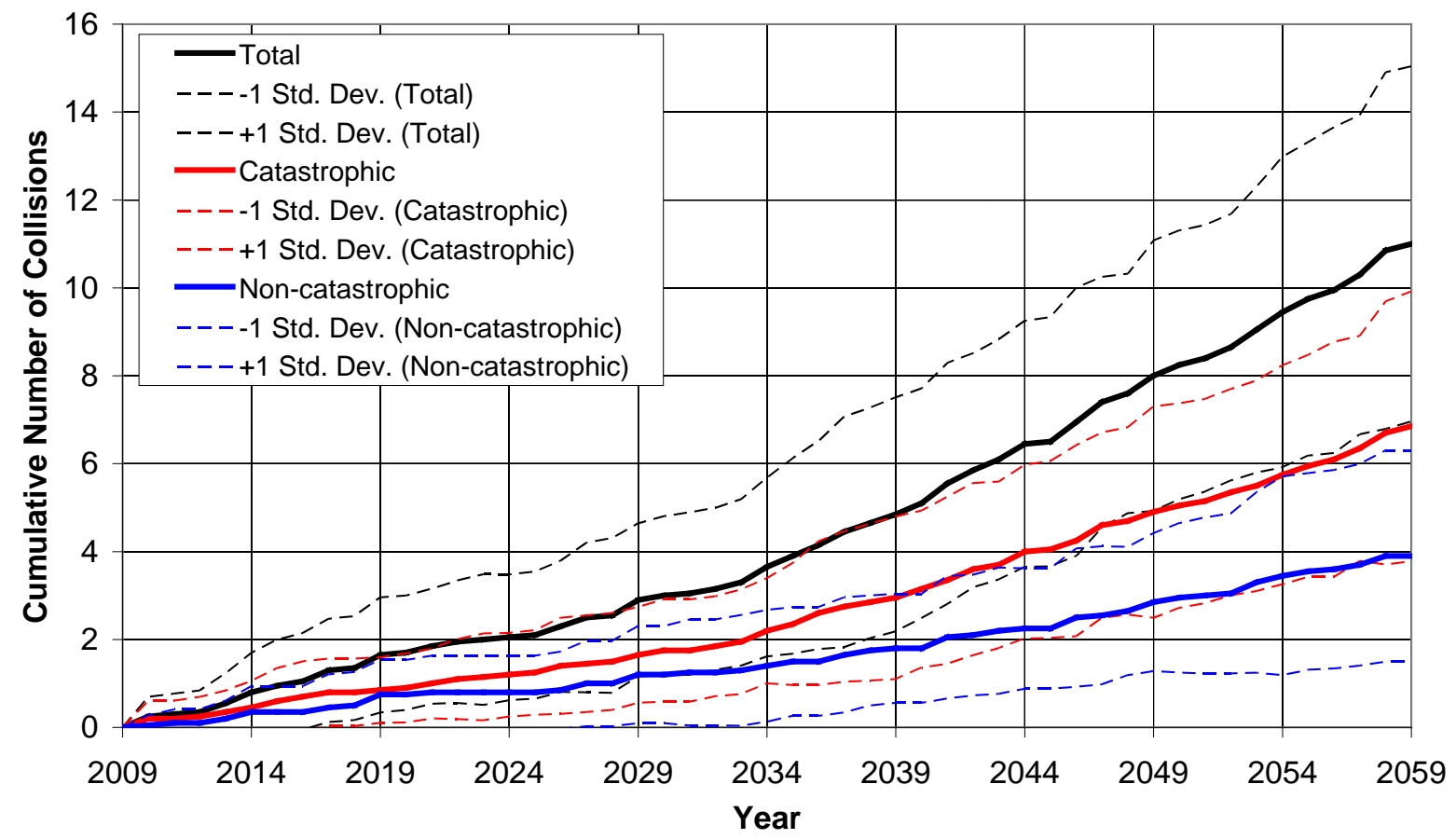


Fig. 17

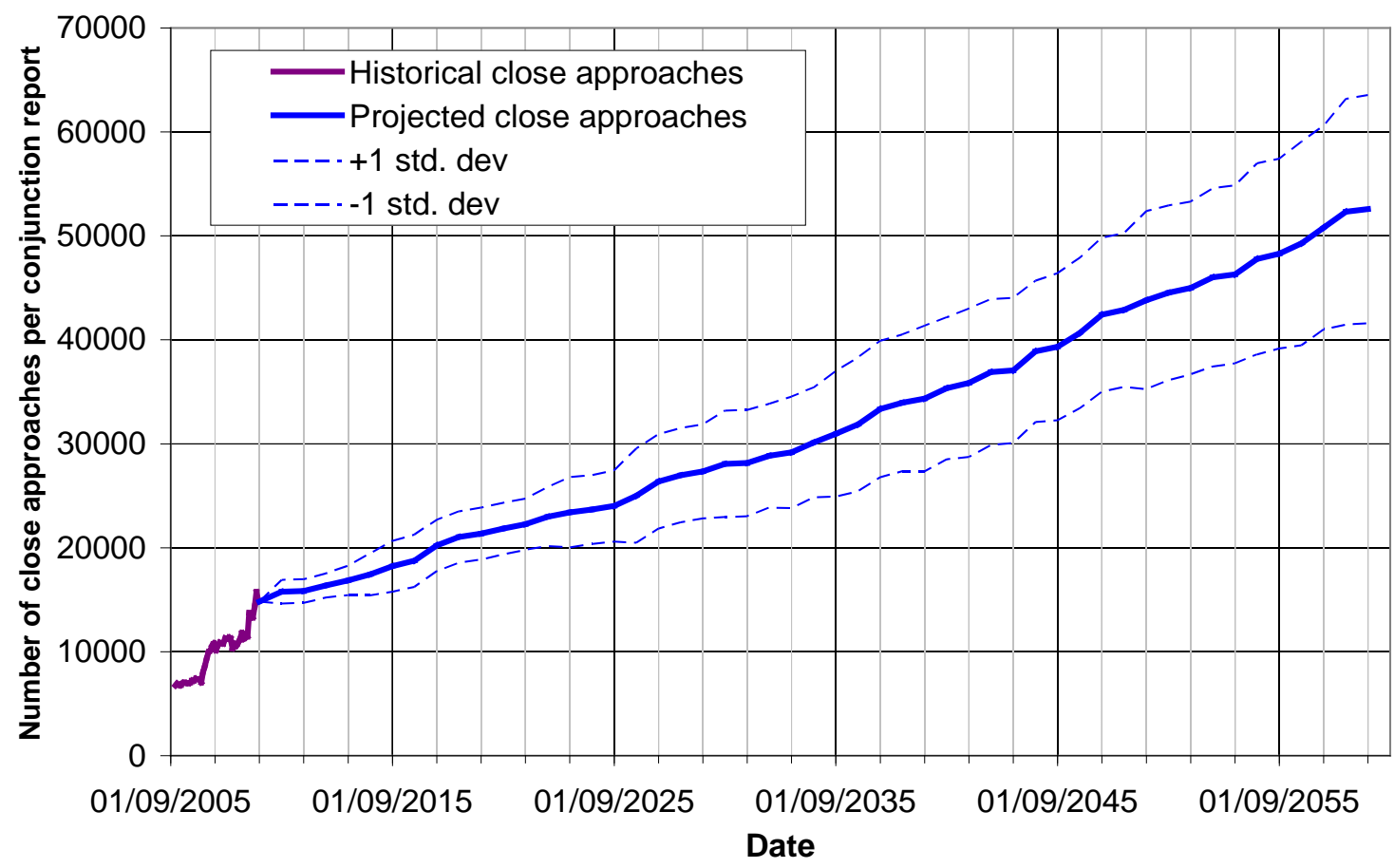


Fig. 18

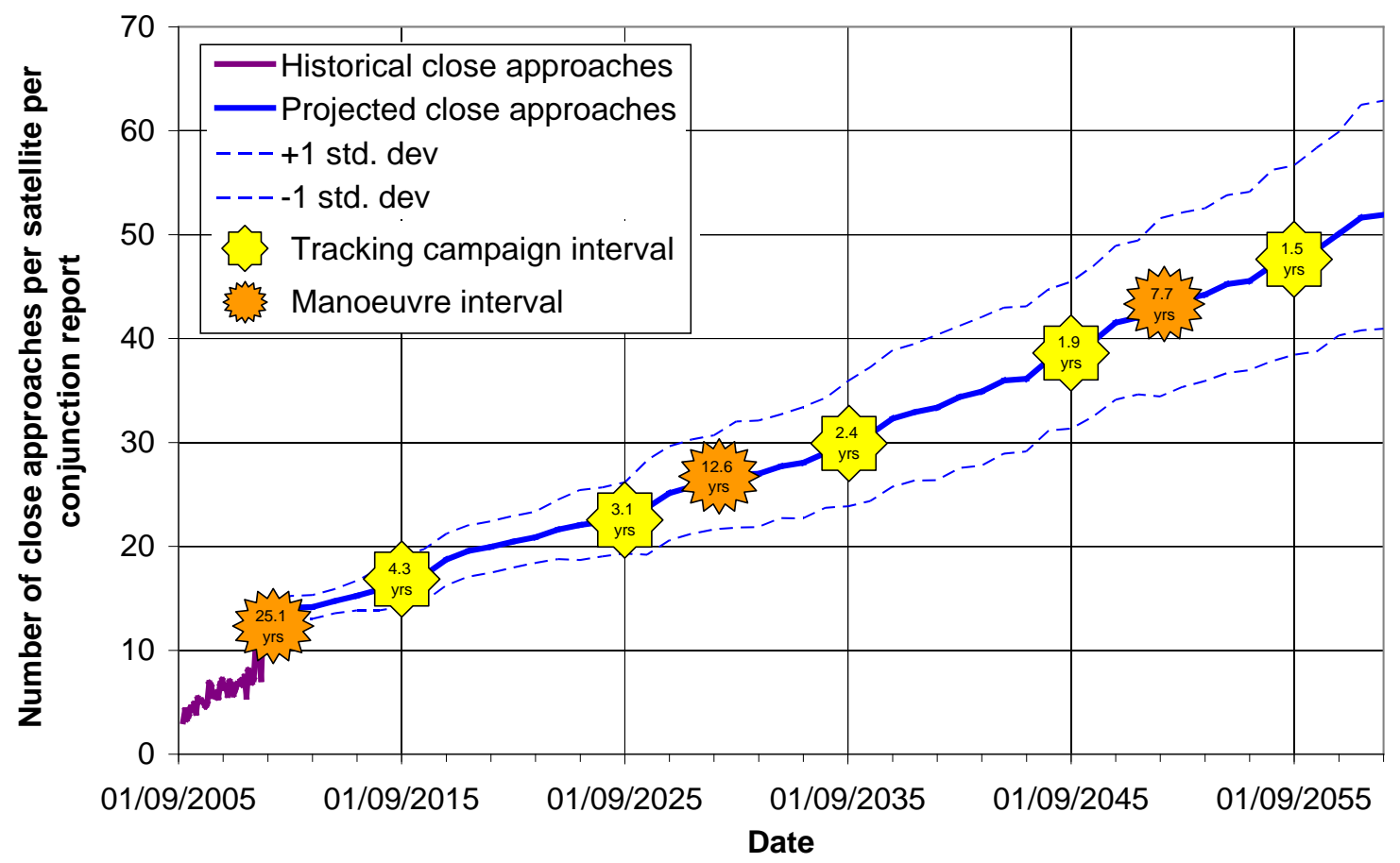

